\title{
Síndrome cistocerebral: a propósito de un caso.
}

\author{
Cystocerebral syndrome: A case report.
}

\author{
Juan Manuel Escobar-Montalvo ${ }^{1,2}$, Erika Herráez-Sanchez ${ }^{1}$, Javier Oliva Navarro ${ }^{1}$, Rosario Torres ${ }^{3}$, \\ Cristian Rízea ${ }^{1,2}$

\section{RESUMEN}

El síndrome cistocerebral fue descrito por primera vez en 1990 por Blackburn y Dunn. Los casos estudiados fueron varones ancianos con síndrome confusional agudo y retención urinaria aguda que, tras un drenaje vesical, presentaron resolución completa del cuadro clínico. Se reporta el caso de un anciano con disminución rápida del nivel de consciencia, mioclonías, hipotensión arterial, bradicardia y retención aguda de orina que experimentó total remisión del cuadro clínico luego del drenaje vesical correspondiente. Se describen, asimismo, los posibles mecanismos implicados en el origen de este síndrome y las alteraciones hemodinámicas y autonómicas subyacentes. Se sugiere considerar al síndrome cistocerebral en el diagnóstico diferencial de pacientes varones ancianos con síndrome confusional o deterioro cognitivo e hipertrofia prostática y que presenten, además, un episodio de retención urinaria aguda.

PALABRAS CLAVE. Mioclonía, anciano, delirio, retención urinaria.

\section{SUMMARY}

Cystocerebral syndrome was first described in 1990 by Blackburn and Dunn, in elderly males with acute confusion syndrome and urinary retention, who after bladder drainage experienced full resolution of the clinical picture. We report the case of an elderly male patient with Cystocerebral syndrome and symptoms such as a rapid decrease in consciousness level, myoclonies, hypotension, bradycardia and acute urinary retention who, after bladder drainage presented a complete remission of the clinical picture. The potential mechanisms involved in the origin of this syndrome are described, as well as its underlying hemodynamic and autonomic alterations. Cystocerebral syndrome should be considered in the differential diagnosis of patients with a confusional syndrome and cognitive impairment, diagnosed with prostatic hypertrophy and presenting, in addition, an episode of acute urinary retention.

KEYWORDS: Myoclonus, aged, delirium, urinary retention.

Sección de Neurofisiología Clínica, Servicio de Neurología, Hospital Universitario La Paz. Madrid, España.

2 Centro de Referencia de Trastornos Complejos del Sistema Nervioso Autónomo, Servicio de Neurología, Hospital Universitario La Paz. Madrid, España.

3 Servicio de Urgencias, Hospital Universitario La Paz. Madrid, España. 


\section{INTRODUCCIÓN}

El síndrome cistocerebral (SCC) fue descrito por primera vez en 1990 por Blackburn y Dunn, en varones ancianos con síndrome confusional agudo y retención aguda de orina, quienes tras el drenaje vesical presentaron la resolución completa del cuadro clínico (1). Posteriormente se han descrito casos de características similares a los que se han añadido otras manifestaciones clínicas como mutismo, tremor, movimientos de características mioclónicas y alteraciones de los mecanismos autonómicos que regulan la tensión arterial y la frecuencia cardiaca (16). En el presente reporte de caso se busca describir un caso típico de un paciente con SCC, así como los factores de riesgo y los potenciales mecanismos asociados a este síndrome.

\section{Caso clínico}

Presentamos a un varón de 86 años que acudió al servicio de urgencias por desorientación en tiempo y espacio, lenguaje incoherente, agitación psicomotriz, movimientos mioclónicos, bradicardia e hipotensión arterial. La instauración de los síntomas clínicos fue progresiva y de unas 12 horas de evolución. El paciente era independiente para actividades de la vida diaria y no se reportó deterioro cognitivo, tenía como antecedentes patológicos hipertensión arterial, hipercolesterolemia, infarto agudo de miocardio, estenosis aórtica leve e hipertrofia prostática benigna. Los fármacos que tomaba de manera crónica eran únicamente ácido acetilsalicílico $100 \mathrm{mg} / 24 \mathrm{~h}$, enalapril $10 \mathrm{mg} / 24 \mathrm{~h}$ y atorvastatina $40 \mathrm{mg} / 24 \mathrm{~h}$. En la exploración física se mostraba somnoliento, presentaba movimientos de características mioclónicas positivas, que se presentaban de forma intermitente, generalizadas de predomino en tronco y extremidades en ambos hemicuerpos de forma simétrica y síncrona. Además, se encontró un soplo sistólico aórtico II/VI, leve distensión abdominal y dolor a la palpación en el hipogastrio. Los análisis de sangre no mostraron alteraciones hematológicas ni bioquímicas significativas y la tomografía cerebral mostró signos leves de atrofia cortico-subcortical difusa y de leucoencefalopatía isquémica crónica de pequeño vaso sin datos de patología aguda, hallazgos similares a los encontrados un año antes en una

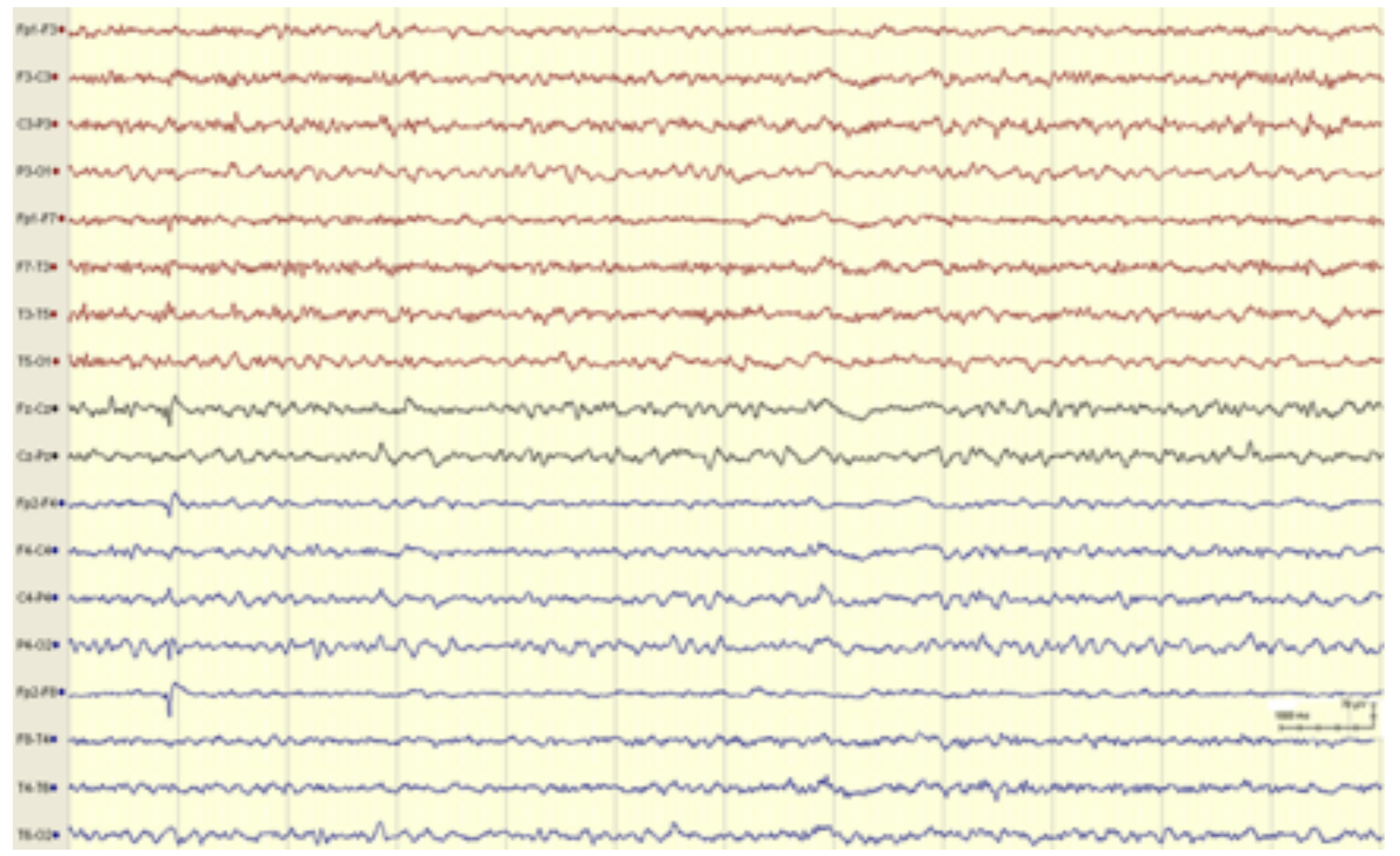

Figura 1. Actividad electroencefalográfica compatible con una encefalopatía de grado leve-moderado, caracterizada por una pobre diferenciación topográfica y constituida por ondas en rango theta con brotes de ondas delta, sin presentar criterios de status epilepticus mioclónico. 




Figura de elaboración propia, diseñada por los autores.

Figura 2. Mecanismos fisiopatológicos posiblemente implicados en el síndrome cistocerebral SCC: Síndrome TAM: Tensión arterial media. EEG: electroencefalograma.

tomografía cerebral realizada tras un traumatismo craneal leve. En la evolución clínica se observó, a las 6 horas de su llegada, un empeoramiento del nivel de consciencia, pasando de un síndrome confusional a un estado de somnolencia con escasa respuesta a estímulos verbales y un aumento de los movimientos mioclónicos. Ante la sospecha de un status epilepticus mioclónico se realizó un electroencefalograma (EEG) urgente, de 30 minutos de duración, en el que se observó una actividad bioeléctrica cerebral continua, con escasa diferenciación topográfica, reactiva a la apertura y cierre ocular, constituida por ondas theta difusas entremezcladas con algunas ondas delta de voltaje medio, sin asociar anomalías epileptiformes ni cumplir criterios de status epilepticus. No se evidenció correlato entre la actividad electroencefalográfica y los movimientos de características mioclónicas, lo cual indicó que estos movimientos sean de origen subcortical. La conclusión del estudio EEG fue compatible con una encefalopatía difusa de grado leve-moderado (figura 1). Además, se observó que a pesar de la administración intravenosa de $\mathrm{NaCl}$ al $0,9 \%$ persistían la bradicardia y la hipotensión arterial. Debido a este empeoramiento se repitió la exploración clínica, encontrándose una mayor distensión abdominal de predominio en hipogastrio por lo que se realizó un drenaje vesical, extrayéndose aproximadamente $1500 \mathrm{ml}$ de orina. Tras unos 20 minutos del drenaje vesical y sin realizar ninguna otra intervención, se observó una muy significativa mejoría clínica. El paciente se encontraba alerta, orientado en espacio y persona, tenías un lenguaje coherente y presentó una remisión completa de los movimientos mioclónicos. Asimismo, la distensión y el dolor abdominal remitieron, y se normalizaron los valores de frecuencia cardiaca y tensión arterial. El resultado del examen de orina obtenido tras el sondaje vesical fue normal. Posteriormente fue evaluado por un especialista en urología quien indicó mantener la sonda vesical al alta y continuar controles de forma ambulatoria. Tras 24 horas de observación fue dado de alta asintomático.

\section{DISCUSIÓN}

El SCC ocurre de forma aguda habitualmente en varones mayores de 70 años, se caracteriza por un síndrome confusional agudo, disminución del nivel de consciencia, agitación y/o temblores, y se asocia en todos los casos con una retención aguda de orina que es seguida por la resolución completa de los síntomas tras el drenaje vesical (1-7). Los factores de riesgo del SCC son edad avanzada, sexo masculino, deterioro cognitivo, infecciones de las vías urinarias, insuficiencia renal, estreñimiento, movilidad reducida e hipertrofia prostática $(5,6)$. 
Los mecanismos propuestos del SCC indican que la distensión mantenida de la pared vesical ocasiona una hiperestimulación del sistema nervioso simpático con aumento de la respuesta beta-adrenérgica y de catecolaminas a nivel central (núcleo coeruleus) y periférico. Este incremento sería el responsable de los síntomas confusionales y de la agitación motora (2, 4-6). Además, se ha observado que algunos pacientes, a pesar de esta activación simpática, presentan hipotensión arterial y bradicardia. Esta respuesta paradójica podría explicarse con los trabajos de Lee y col., quienes observaron que la distensión mantenida de la vejiga urinaria en pacientes con hiperlipidemia se relaciona con vasoconstricción epicárdica e incremento de las resistencias coronarias $(7,8)$. Esto disminuiría la contractilidad y la fracción de eyección cardiaca, con una reducción de la frecuencia cardiaca, de la tensión arterial media y del gasto cardiaco, que podría ocasionar secundariamente una reducción de la perfusión cerebral y un enlentecimiento de la actividad eléctrica cortical vista en el EEG (9). Adicionalmente, otro factor que contribuiría con este fenómeno es la desensibilización beta-adrenérgica que consiste en la reducción de la capacidad inotrópica positiva del ventrículo izquierdo tras un estímulo adrenérgico asociado al envejecimiento (figura 2) (10). Estas hipótesis podrían explicar la presentación, la evolución y la resolución completa del cuadro clínico del paciente tras el drenaje vesical. Finalmente, se sugiere tras descartar otras causas potencialmente graves o letales, incluir el SCC dentro del diagnóstico diferencial de pacientes con síndrome confusional o delirium que tengan edad avanzada, alteración aguda del nivel de consciencia, factores de riesgo de SCC y un examen clínico compatible con una retención aguda de orina (11).

\section{Correspondencia:}

Dr. Juan Manuel Escobar Montalvo

Correo electrónico: juanmaes@ucm.es

\section{REFERENCIAS BIBLIOGRÁFICAS}

1. Blackburn T, Dunn M. Cystocerebral syndrome. Acute urinary retention presenting as confusion in elderly patients. Arch Intern Med. 1990;150(12):25778. doi: 10.1001/archinte.150.12.2577

2. Blè A, Zuliani G, Quarenghi C, Gallerani M, Fellin R. Cystocerebral syndrome: a case report and literature review. Aging (Milano). 2001;13(4):33942.

3. Waardenburg IE. Delirium caused by urinary retention in elderly people: a case report and literature review on the "cystocerebral syndrome". J Am Geriatr Soc. 2008;56(12):2371-2.

4. Saga K, Kuriyama A, Kawata T, Kimura K. Neurogenic bladder presenting with cystocerebral syndrome. Intern Med. 2013;52(12):1443-4.

5. Shirvani N, Jimenez XF. Cystocerebral Syndrome: A Case Report and Review of Literature and Mechanisms. J Am Geriatr Soc. 2015; 63(12): 26452647.

6. Liem PH, Carter WJ. Cystocerebral syndrome: a possible explanation. Arch Intern Med. 1991;151(9):1884-1886.

7. Thelmo F, Tzarnas S, Rosal N, Kramer M, Walters L. Cystocerebral Syndrome: An Updated Review and a New Proposed Mechanism for an Often Forgotten Cause of Delirium. Cureus. $2020.19 ; 12(10)$ :e11034.

8. Lee TM, Su SF, Chen MF, Tsai CH. Acute effects of urinary bladder distension on the coronary circulation in patients with early atherosclerosis. J Am Coll Cardiol. 2000;36(2):453-60. doi: 10.1016/s07351097(00)00751-8

9. Tsung-Minglee L, Sheng-Fang S, Chang-Her Tsai. Effects of distension of urinary bladder on coronary conduit and resistance vessels in hyperlipidemic patients. Clin. Cardiol. 2002;25:467-473.

10. Vincent J. Understanding cardiac output. Critical Care. 2008;12:174. doi: 10.1186/cc6975

11. Ferrara N, Komici K, Corbi G, Pagano G, Furgi G, Rengo $\mathrm{C}$, et al. $\beta$-adrenergic receptor responsiveness in aging heart and clinical implications. Front Physiol. 2014;4: 396. doi: 10.3389/fphys.2013.00396

12. Marcantonio E. Delirium in Hospitalized Older Adults. N Engl J Med. 2017;377(15):1456-1466. doi: 10.1056/NEJMcp1605501

Recibido: 10/02/2021

Aceptado: 03/05/2021 